\title{
Using Near Field Communication Technology to Achieve Near-Zero-Configuration of Sensors
}

\author{
Nicolaj Bjerregaard Christensen \\ Engineering College of Aarhus \\ Dalgas Avenue 2 \\ DK8000 Aarhus C, Denmark \\ +4587302423 \\ nbc@mjolner.dk
}

\author{
Stefan Wagner \\ Engineering College of Aarhus \\ Dalgas Avenue 2 \\ DK8000 Aarhus C, Denmark \\ +4587302423 \\ sw@iha.dk
}

\begin{abstract}
Numerous companies and educational institutions have begun research into pervasive healthcare systems to address the growing need for caretaking and out-of-hospital monitoring. These systems allow for automatic and distributed monitoring of patients, but inherently require installation and maintenance configuration of the sensor network and communication platform. To avoid burdening caretaking staff and relatives with installing and configuring system software and hardware, a research project at the Engineering College of Aarhus, Pervasive Healthcare lab, has been trying to achieve a zero-configuration solution, removing most handling while at the users home. Achieving a true zero-configuration strategy has proven hard to implement especially with regards to the coupling of the individual wireless sensors to a specific patient. This paper describes a prototype solution designed to overcome these problems using near field communication technology, which appears to be a promising solution.
\end{abstract}

\section{Categories and Subject Descriptors}

H5.2 [Information interfaces and presentation]: User interfaces -input devices and strategies.

\section{General Terms}

Human Factors, Design

\section{Keywords}

Pervasive healthcare, NFC, HCI, ambient assisted living, ubiquitous computing, zero-configuration, sensors, wireless

\section{INTRODUCTION}

Pervasive healthcare is an ever-growing industry and research area, which has attained great attention during the last decade [1].

Permission to make digital or hard copies of all or part of this work for personal or classroom use is granted without fee provided that copies are not made or distributed for profit or commercial advantage and that copies bear this notice and the full citation on the first page. To copy otherwise, or republish, to post on servers or to redistribute to lists, requires prior specific permission and/or a fee. MobiQuitous 2008, July 21-25, 2008, Dublin, Ireland. Copyright @ 2008 ICST ISBN 978-963-9799-27-1
Especially out-of-hospital monitoring, in areas such as post-release monitoring, pre-submission monitoring and monitoring the elderly has been major focus areas.

Monitoring patients post-release, especially heart-failure patients, have been shown to be successful and allows hospitals, and ultimately the society as a whole, to save time and money on re-admissions [1]. and caretakers to monitor released patients continuously.

This can be achieved through the use of personal sensors and a manual or automatic data submission system, which will allow doctors Pre-submission monitoring, where patients in certain danger groups are being monitored, e.g. patients with high blood pressure, and post-release monitoring have also been shown to improve not only the quality of life of the patients, but also the general health of them [3],[4]. Again through personal sensor networks, the patient is able to submit health data to doctors automatically and receive a warning or message whenever dangerous readings occur.

Monitoring the elderly is an equally large subject, which can help improve the quality of life of the elderly. This is achieved by utilizing pervasive sensor networks. Providing continuous monitoring of an elderly with a heart condition can provide security for the patient and the family, knowing that any dangerous readings can be responded to immediately [5]. Pervasive networks also allow monitoring of non-medical readings, such as falling, lack of exercise, amount of medicine taken etc.

All of these sensor networks have similar requirements. A large number of sensors, depending on the illness and the general situation of the patient, may be required. Configuring and using these sensor networks can be cumbersome or even impossible to do for the patient. This is especially true when using manually operated sensors or when monitoring non-technical patients, such as many elderly patients.

Operating mobile phones, automatic pill dispensers or even touch screens might not be feasible for some patient groups, and the solution is to automate as much as possible. Embed the sensors into everyday items and allow the 
sensors and monitoring stations to collect data automatically. User interaction can be held at a minimum, without taking away control from the patient. Pervasive healthcare lets us install large sensor networks with a minimum of interference with the everyday life of the patient.

One common problem with many pervasive sensor networks, especially in densely populated areas, is automatic setup of sensors and monitoring stations. Using Bluetooth or $\mathrm{WiFi}$, the ranges of units will overlap causing confusion with unit pairing when using zero-configuration setups where the units pair and configure automatically, as shown in Fig 1.

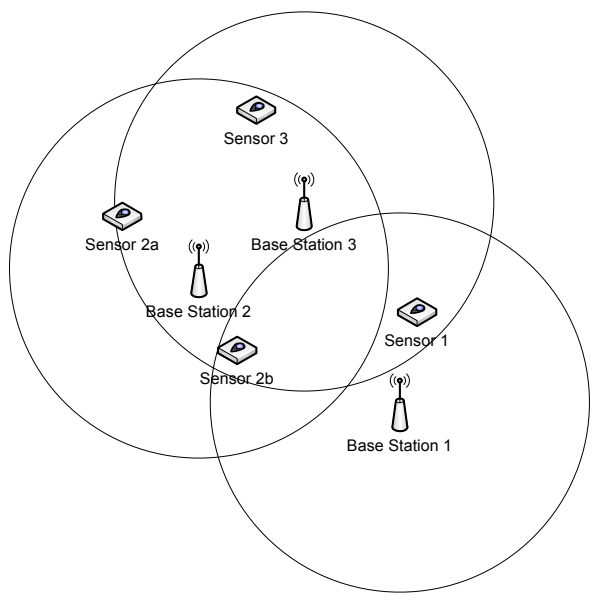

Fig. 1 Overlapping sensors and base stations

A common solution is to have the patients themselves, or their relatives, configure the units, either entirely manual or by supplying them with preconfigured units. The preconfigured units must then be manually setup by caretakers and technical staff.

Another solution is to have the caretaking staff configure the units on the go, taking up valuable time that could be better spent helping or nursing the patients.

\section{EXISTING FRAMEWORK}

An example of a Pervasive Healthcare framework is the Healthcare@Home system [6] developed at the Engineering College of Aarhus. This system contains a zeroconfiguration sensor framework, which allows sensors and base stations to configure and pair automatically. This is accomplished through Bluetooth, $\mathrm{WiFi}$ and other wireless and wired technologies. The system today supports a wide range of wireless sensor types from different manufactures; blood-pressure, heart rate, ECG, oximeter, weight and blood-sugar devices, and all of these may be automatically detected, download and activated in a running system in the users home, without any kind of user interaction, hence achieving zero-configuration.

The system is functional, but while there are no sensor range overlap issues in isolated installations, the currently constructed framework architecture proved to suffer from the earlier mentioned problems when several patients are within range of each others base stations: The zeroconfiguration units are unable to determine which sensor belongs to which patient and base station, in case of these not being either preconfigured or configured by manual onsite user interaction, thus breaking the zero-configuration paradigm.

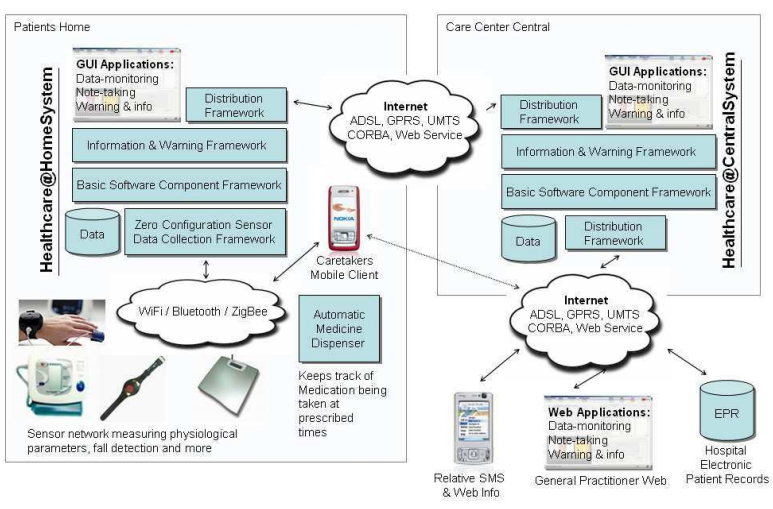

Fig. 2 The existing Healthcare Infrastructure, with zero-configuration capabilities. Data is automatically collected from all the wireless sensors and distributed to the authorized stakeholders.

\section{PROPROSED SOLUTION}

A number of different solutions where considered for overcoming the issue of overlapping sensors and base stations, including voice recognition software for verification of the identification of a new wireless sensor to the system, over to a simple caretaker initiated touch-screen soft-key-press-verification when introducing a new sensor. All of these solution decreases the usability of the originally intended zero-configuration strategy of the project, as well as having a lower level of security and thus a higher risk of failure. Both of the mentioned solutions have been planned for future projects to be tested along with the proposed solution.

In this paper, we present a third solution that has been developed which attempts to address the above mentioned setup and configuration issues with pervasive sensor networks, by utilizing near field communication (NFC) enabled mobile phones [7]. This solution will in time be a part of the Healthcare@Home framework and will allow end users, such as nurses, caretakers or even patients themselves, to pair sensors and base stations easily and quickly without requiring any technical skills. 
This NFC based solution adds another communication interface to each of the sensors, the base station and a patient proxy. It utilizes attaching NFC tags to each sensor and base station. These tags contain basic information about the unit, such as type and ID (MAC address or social security number). The implemented application, SIH Sensor Pairing Application (SIHSPA), will allow users to pair units by simply touching the NFC tags with their mobile phone and automatically upload the pairing data to the central server, as illustrated in Fig 3.

By using NFC, instead of more widely used short-range communication technologies such as Bluetooth, it is possible to perform the pairing process automatically, without requiring the user to enter any kind of ID, unit type, passkey or similar information. NFC-enabled Javasupporting cell phones allow automatic application start whenever an NFC tag is read (which occurs upon contact), which minimizes the required user interaction.

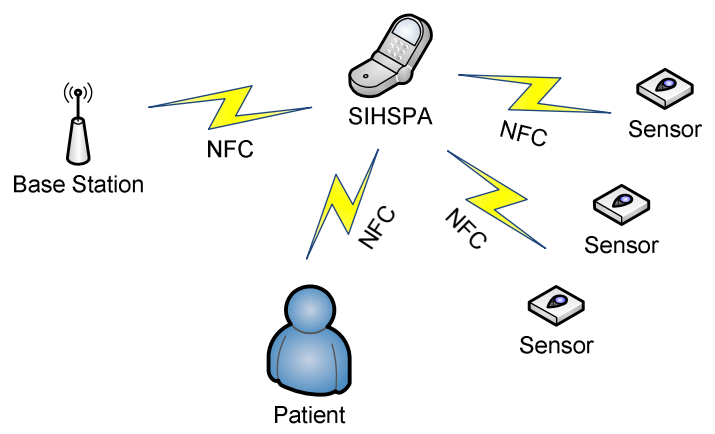

Fig. 3 Communicating via NFC

Furthermore, by integrating the NFC solution on a mobile phone most users will be familiar with the user interface. While the ability of most modern phones to communicate over the internet, will allow the user to upload information immediately. Figure 4 shows the architecture of the mobile to server communication.

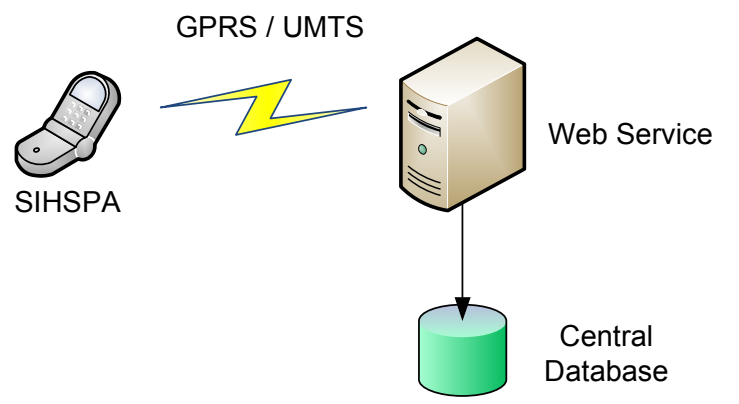

Fig. 4 Uploading pairing data to the central database

\section{DESIGN}

The application has been implemented as a Java ME Midlet. It has been build using the Nokia Software Development Kit for Nokia 6131 NFC 1.1 [8].

By designing it as a Java ME Midlet the application is able to run on any Java enabled phone which supports the Contactless Communication API (JSR-257). Currently there is only a very limited number of NFC enabled mobile phones available, which limits the choice of mobile phones. The application can, because of the inherent multi-platform features of Java, easily be converted to using a more generic SDK, as it becomes available in time. It may also be considered porting the application to Linux [9] or the Windows Mobile platform.

The design has mainly focused on creating an easy-touse interface that will allow anyone to use the application. It has been considered imperative that the application would not become frustrating or counterproductive to use, that no screens or messages are ambiguous and that the user feels the application helps and guides without controlling the workflow.

For this reason, a simple text based interface was implemented, with easy to read messages and a simple user interface. With clearly marked next steps and exits, the interface guides the user along the pairing process and allows the user to abort at any time. See Fig. 5 for an example of the user interface.

Behind the interface, the application was designed to be responsive and intuitive to use. Including only minimal wait time when the application is started automatically by the user touching a sensor with the phone and thereby initiating the reading of the sensor's NFC tag.

Upon manual program start the user will be greeted with an options menu, which will let the user either activate automatic start (only needs to be done once) or remove an already established sensor pairing. As seen in Fig. 5, the interface is easy to understand and has clearly marked options and exits. The options at the bottom correspond to the three physical buttons below the screen (not shown).

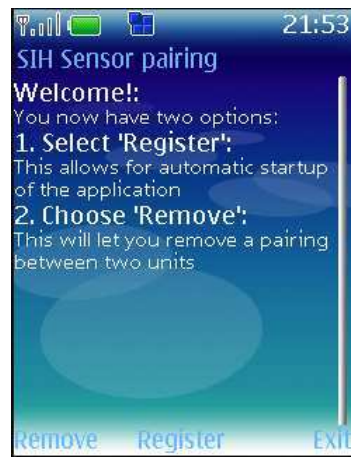

Fig. 5 The main menu of the application 
Upon automatic start when an NFC tag has been detected, the application displays a screen which shows the type of tag found (sensor, base station or patient) along with the required type of tag to touch next. This serves a guiding purpose towards completing the pairing process. Again there is a clearly marked exit in case the user mistakenly touched a tag. The screen can be seen in Fig. 6 (left).

Figure 5 (right) shows the user confirmation that is displayed upon detecting the correct second tag. To ensure that the wrong units are not paired, the types of tags detected are displayed along with 'Yes' And 'No' options. If the user selects the 'Yes' option, the pairing data is automatically uploaded to the server and the rest of the system can begin using the information immediately.
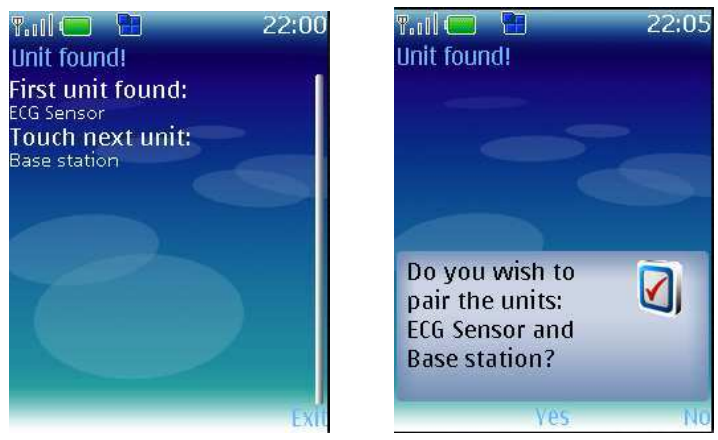

Fig. 6 Left: shows the first tag has been found. Right: lets the user choose whether to pair the two units found.

Furthermore, the application will allow pairing between base stations and patients, to configure which patient the sensors paired to the base station will collect data for. This pairing process is the same as the one described above between sensors and base stations. The patient will be given an NFC tag that contains basic patient information such as social security number, which can be placed somewhere in the patients' home, in a keychain or similar. The patient tag can be embedded in a simple box or accessory depending on the patient.

\section{NFC INTRODUCED}

Near Field Communication (NFC) is a standardsbased, short-range (from a few centimeters to physical contact) wireless connectivity technology that enables simple and safe two-way interactions between electronic devices. For instance enabling two cell phones to exchange Bluetooth MAC addresses and pairing keys, for easy coupling of the devices using Bluetooth afterwards. Or a cell phone to easily couple with a Bluetooth headset, without having to go through the manual process of pairing the two devices. NFC was thus developed for pairing wireleses devices with a minimum configuration effort, and thus solving one of Bluetooths major shortcomings, namely the manual pairing process, involving different steps, including device discovery, key exchange, and pairing. By using proximity as primary security feature, this technology might be vulnerable on its own, but not when utilizing a server component as in this propsed solution.

The Nokia 6131 NFC phone (see figure 7) is equipped with NFC capability - and may be configured to start a Java application upon touching a tag - and automatically transfer data between tag and phone. This makes it possible to obtain context specific information in a minimum-userinteraction scenario.

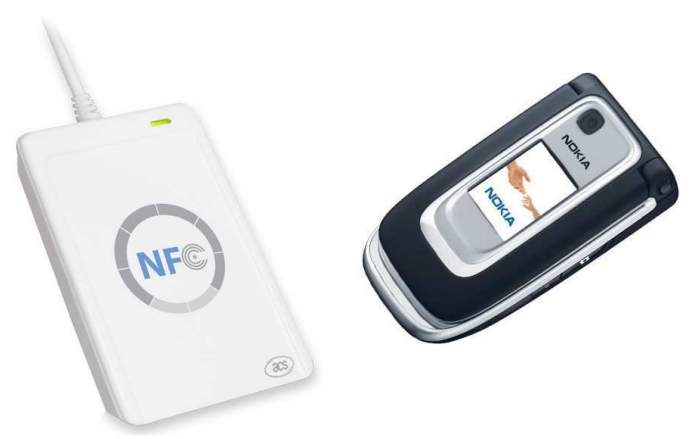

Fig. 7 There are several NFC readers available as- including the above ACR122 NFC Contactless Smart Card Reader (shown next to the Nokia 6131 NFC phone, used in this project)

NFC is supported by a wide range of commercial companies and this might result in a wider range of products supporting NFC technology being released in the near future. NFC is also planned integrated in the open source pervasive healthcare project, OpenCare Project [10].

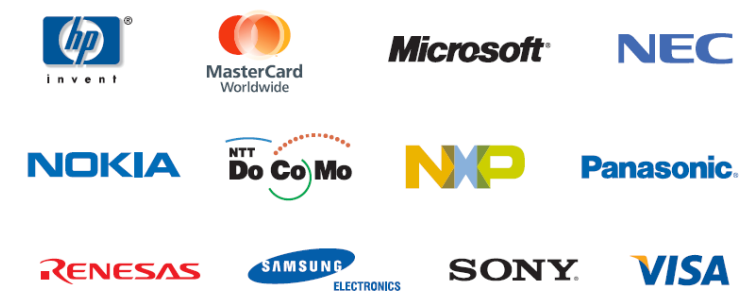

Fig. 8. NFC is backed by a wide range of software and hardware manufactures. Only a few products are available

\section{RESULTS}

A prototype Java MIDP NFC application has been constructed which is able to read the NFC tags of sensors, base station and patients, and couple these by calling a web service over GPRS. It has not yet been fully implemented in 
the Healthcare@Home infrastructure, but this is work in progress.

The application solves the problem of sensor range overlaps outlined in previous sections of this paper, by adding an extra near-zero-configuration element to the existing Healthcare@Home framework. In areas where sensors might overlap, the caretakers are able to pair, and un-pair, sensors and base stations easily and efficiently without having any technical knowledge of the framework itself. The ability to pair a specific base station with a specific patient will further ease the setup process, as multiple base stations can be placed in close proximity without fear of pairing problems between base stations and sensors.

\section{DISCUSSION \& FUTURE WORK}

The proposed solution appears to have the potential to solve most of the issues with sensor pairing, but it is still a fair distance from zero-configuration. The current requirement to use Nokia NFC phones, due to lack of other models on the marked, is not an ideal solution, but for now, we must wait for more models. Furthermore, in Denmark, some municipalities are for instance using Windows Mobile powered devices as their caretaking staffs communication platform. These user groups should not be forced to use two models of communication devices, so we need to support all popular mobile platforms used today.

Even though simply touching a number of sensors, a base station and a patient, does not appear to be a cognitive challenging task we do not know whether the solution is user friendly or not. Only after having completed thorough user studies can we be certain, that the solution is in fact user friendly. Also, the planned alternative user interface, including speech recognition for verification or touch screen based interaction, should also be tested with the users. Usability tests are planned in the near future.

As an alternative to using NFC enabled Java cell phones, the Healthcare@Home framework could be modified so that the base stations contained an NFC reader instead of just a tag (see figure 7, right). This way the caretakers or the patients themselves, could pair a sensor with a base station simply by touching the base station with the sensor (which would still contain an NFC tag). The application could then be used to remove already setup pairings, read information from tags, read patient records, pair patients and base stations or similar miscellaneous operations. This would result in a simpler workflow and allow for easy patient interaction, as well as remove the mobile phone from the pairing process, which might help some elderly patients feel safer about it.

\section{CONCLUSION}

A solution for pairing wireless sensors to a base station and a patient has been proposed, and a working prototype has been successfully implemented, using NFC technology on a Nokia Java equipped cell phone.

The solution has not yet been integrated with the Healthcare@Home framework, but the framework database and infrastructure has already been prepared for this, and implementation is planned in the near future.

Whether the solution will appear user friendly to the caretaking staff, who are meant to be using them, and whether it will provide true near-zero-configuration functionality or not, might only be proven through usability experiments and field trials.

\section{REFERENCES}

[1] J.E. Bardram, A. Mihailidis, D. Wan, "Pervasive Computing in Healthcare", CRC Press (2006).

[2] J. Morak, A. Kollmann, D. Hayn, P. Kastner, G. Humer, G. Schreier, "Improving Telemonitoring of Heart Failure Patients with NFC Technology", Proceedings of the $5^{\text {th }}$ IASTED International Conference, Innsbruck, Austria (2007).

[3] F. Gouaux, L. Simon-Chautemps, S. Adami, M. Arzi, D. Assanelli, J. Fayn, M.C. Forlini, C. Malossi, A. Martinez, J. Placide, G.L. Ziliani, P. Rubel, "Smart Devices for the Early Detection and Interpretation of Cardiological Syndromes", Proc of the 4th Annual IEEE Conf on Information Technology Applications in Biomedicine, UK.

[4] Teknologirådet, "Sundhedsydelser med IT - Pervasive Healthcare i den danske sundhedssektor", Teknologirådets rapporter 2006/11, p. 61-64.

[5] IBM, " ElderTech - interaktive ældreboliger", [Online], Available: http://www.teknikogviden.dk/artikler/visArtikel.asp?id=6421

[6] Stefan Wagner, "Towards an Open and Easily Extendible Home Care System Infrastructure", Proceedings of the $2^{\text {nd }}$ International Conference on Pervasive Computing Technologies for Healthcare, Tampere, Finland (2008).

[7] NFC Forum, "Near Field Communication and the NFC Forum", [Online], Available: http://www.nfcforum.org/resources/white_papers/nfc_forum_marketing_wh ite_paper.pdf (04.01.2008)

[8] Nokia, "Nokia 6131 NFC SDK 1.1", [Online], Available: http://www.forum.nokia.com/info/sw.nokia.com/id/ef4e1bc9 -d220-400c-a41db3d56349e984/Nokia_6131_NFC_SDK.html (04.01.2008)

[9] Yaw Y. Anokwa, "Beyond Device Pairing: New Interactions on NFC Enabled Mobile Phones", Department of Computer Science and Engineering, University of Washington (2007).

[10] Wagner, S., "OpenCare Project Infrastructure”, [Online], Available: http://opencareproject.com (04.01.2008) 